\title{
Performance Comparison and Selection of Transformer Fluid
}

\author{
Yang LU ${ }^{1}$, Shi Jia LIU ${ }^{2}$ \\ Locomotive \& Car Research Institute, China Academy of Railway Sciences, Beijing, China \\ Beijing Zongheng Electro-Mechanical Technology Development Co , Beijing, China
}

\begin{abstract}
Transformer fluid directly affects the working state of the components and the cooling efficiency of transformer. There are three kinds of transformer fluid used for electric locomotive, EMU and suburban rail vehicles: mineral oil, silicone liquid and synthetic ester based insulating oil. In this paper, the three kinds of oil are compared from the fire safety, environmental protection, reliability and low maintenance. It provides a strong basis for the selection of transformer fluid. By comprehensive analysis, synthetic ester based insulating oil can completely replace mineral oil and silicone liquid. With rail transport safety and environmental protection standards improving, synthetic ester based insulating oil will be the best choice for transformer.
\end{abstract}

\section{Introduction}

Traction transformer is an important equipment of electric locomotive and EMU, its cooling mode is by insulating oil. Transformer fluid directly affects the working state of the components and the cooling efficiency of transformer. Therefore, the choice of transformer fluid is essential.

Currently, there are three transformer fluid used in electric locomotive and EMU: mineral oil, silicone liquid and synthetic ester based insulating oil (synthetic ester). These three kinds of insulating oil can all meet the basic performance requirements of transformer fluid, but with the rapid development of rail transport technology, it puts forward higher requirements on the performance of transformer fluid. Meanwhile, safety and environmental protection must be considered in the selection of transformer fluid.

In the following article, three kinds of transformer fluid are compared from fire safety performance, environmental performance, reliability and low maintenance. The results can provide a strong basis for the selection of transformer fluid.

\section{Fire Safety Performance}

It is the most suitable transformer cooling method to use insulating oil (not other cooling medium) radiating heat, but this approach also determines that there is a fire hazard for transformer. In the field of rail transportation, the transformer fire is absolutely not allowed to happen. The fire will spread with terrifying speed, and result in a huge human and property losses. Fire safety performance of transformer fluid can be measured from the fire danger grade and ignition-resistant properties.

\subsection{Fire Danger Grade Comparison}

Fire performance comparison result of mineral oil, silicone liquid and synthetic ester is shown in Table 1.Among them, three indicators of mineral oil are the lowest, ignition point of silicone liquid is the highest, other indicators of silicone liquid are the same with synthetic ester. 
Table 1 Fire Performance Comparison Result

\begin{tabular}{|c|c|c|c|c|}
\hline Parameter & $\begin{array}{c}\text { Test } \\
\text { Method }\end{array}$ & $\begin{array}{c}\text { Mineral } \\
\text { Oil }\end{array}$ & $\begin{array}{c}\text { Silicone } \\
\text { Liquid }\end{array}$ & $\begin{array}{c}\text { Synthetic } \\
\text { Ester }\end{array}$ \\
\hline Flash Point & ISO 2719 & $150^{\circ} \mathrm{C}$ & $260^{\circ} \mathrm{C}$ & $260^{\circ} \mathrm{C}$ \\
\hline Ignition Point & ISO 2512 & $170^{\circ} \mathrm{C}$ & $>350^{\circ} \mathrm{C}$ & $316^{\circ} \mathrm{C}$ \\
\hline $\begin{array}{c}\text { Fire Danger } \\
\text { Grade }\end{array}$ & IEC 61039 & $\mathrm{O}$ & $\mathrm{K} 3$ & $\mathrm{~K} 3$ \\
\hline
\end{tabular}

\subsection{Ignition-resistant Properties Comparison}

Place the oxy-acetylene torch flame (over $2000^{\circ} \mathrm{C}$ ) to the fluid surface in the metal pan, thermocouple is placed at the bottom of the dish to detect more distal surface temperature of the oil. Once the torch lit, began to record the oil temperature. Ignition-resistant properties comparison result of synthetic ester and mineral oil is shown in Figure 1. Through experiment observing, the surface of mineral oil began to burn in the first 3 minutes, there is not any change on the surface of synthetic ester in the first 3 minutes, even in the 70th minute no spark appears, shown in Figure 2.

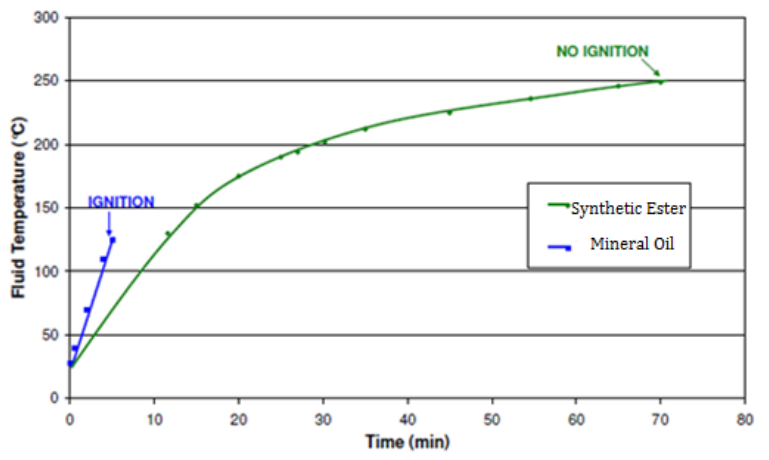

Fig 1 Ignition-resistant Properties Comparison Result

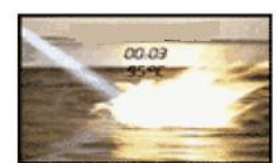

Mineral Oil $3 \min$

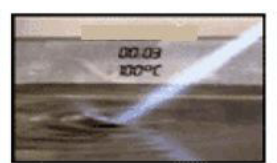

Synthetic Ester $3 \mathrm{~min}$

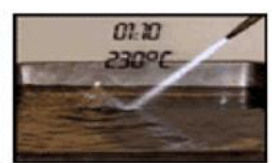

Synthetic Ester $70 \mathrm{~min}$
Fig 2 Burning Test Phenomenon Comparison Result

In addition, synthetic ester passes through the following third-party fire certification:

- Underwriters Laboratory (UL® Classified) - EOUV

Dielectric Medium Classification

(UL fire certification)

- $\quad$ Factory Mutual (FM $\left.{ }^{\circledR}\right)$ Global - FM Approved Less

Flammable Transformer Fluid
(FM fire certification)

- SGS BASEEFA, UK - ATEX Certification (Use authentication in potentially explosive atmospheres)

Thus, the higher fire safety performance of transformer fluid can greatly improve the safety performance of rail transportation equipment.

\section{Environmental Performance}

In the field of rail transportation, the selection of vehicle material is related to the survival of the human environment, transformer fluid is no exception. It could not be ignored how to deal with waste replacement fluid and unexpected leakage fluid. The main factors to judge the environmental performance of transformer fluid are biodegradability, the risk of water pollution and the influences on aquatic life.

\subsection{Biodegradability Comparison}

Biodegradation is a procedure that organic matter is decomposed and made harmless, which can be accepted by environment. Synthetic ester is non-toxic and easily biodegradable, its biodegradability has passed the standard test by laboratories officially recognized abroad. The biodegradation process of synthetic ester is shown in Figure 3.The degradation rate is $10 \%$ at the third day, $71 \%$ at 10 th day, $89 \%$ at 28 th day. This result meets the OECD biodegradable class requirement and the fully biodegradable requirement in IEC 61039 standard.

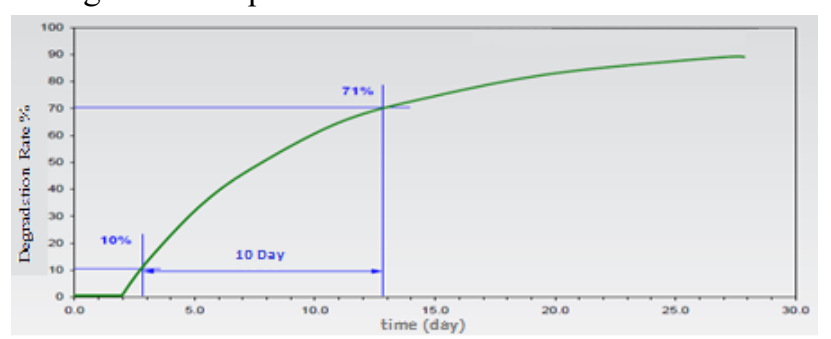

Fig 3 Biodegradation Process of Synthetic Ester

The biodegradation process of mineral oil and silicone liquid is shown in Figure 4. It is obvious that the degradation rates of both mineral oil and silicone liquid are less than $10 \%$ at the end of the test period 28 days, which makes a sharp contrast with synthetic ester. 


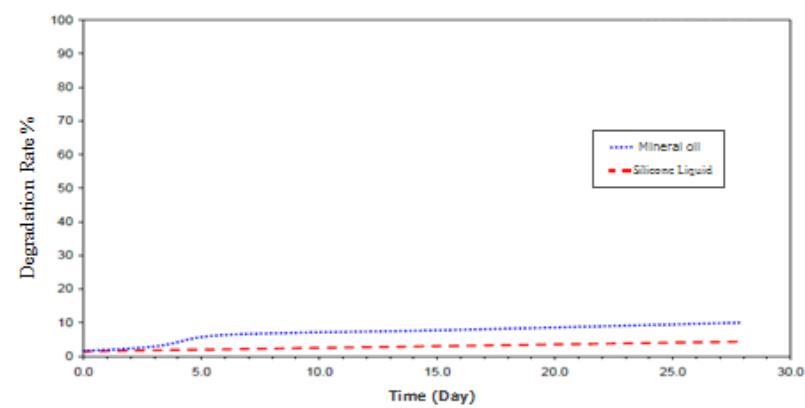

Fig 4 Biodegradation Process of Mineral Oil and Silicone Liquid

\subsection{Risk of Water Pollution Comparison}

Umwelt Bundes Amt(UBA) assess chemicals and divide levels based on biodegradability and the potential impact on aquatic organisms, synthetic ester is classified as non hazardous to water products. Mineral oil and silicone liquid are classified as hazardous to water classes, and the specific test parameters and reference limits are shown in Table 2. Mineral oil and silicone liquid will damage environment. It was confirmed that synthetic ester leaks into river will not cause harm to aquatic organisms at very high concentrations $(1000 \mathrm{mg} / \mathrm{l})$. Once mineral oil or silicone liquid occurs event of leakage it would cause serious environmental pollution, and this effect will not be eliminated in many years.

Table 2 Test Parameters and Reference Limits

\begin{tabular}{|c|c|c|}
\hline Insulating oil & CAS number & UBA classification \\
\hline mineral oil & manifold & 1 (hazardous to water) \\
\hline silicone oil & $63148-62-9$ & 1 (hazardous to water) \\
\hline synthetic ester & $68424-31-7$ & $\mathrm{Nwg}$ (non hazardous to water ) \\
\hline
\end{tabular}

In addition, synthetic ester also passed the following stringent international environmental certification:

- OECD Biodegradable Environmental Report

- UBA Environmental Report on non water pollution

- US government Water Environmental Report

Therefore, synthetic ester owns an obvious advantage of environmental performance, environmental pollution will not be worried.

\section{Reliability}

The main components of the transformer are immersed in transformer fluid. The reliability of transformer fluid directly impacts transformer normal work. It can not only slow down the deterioration rate of fluid, but also reduce the failure rate of the components of the transformer by improving the reliability of transformer fluid. The reliability of transformer fluid is reflected by moisture resistance and lubrication property.

\subsection{Moisture Resistance Comparison}

Moisture resistance of transformer fluid is reflected by water content. With moisture content increasing, the performance of transformer oil will decline, mainly in the following points:

1) Increased water content will decrease dielectric strength.

The breakdown voltage and the water content of synthetic ester, mineral oil and silicone liquid at $20{ }^{\circ} \mathrm{C}$ is shown in Figure 5, Obviously, the water content of mineral oil and silicone liquid is very low, and the breakdown voltage decreases rapidly with the water content increasing. In contrast, the water content of synthetic ester is much larger than mineral oil and silicone liquid, even in the case of the water content over 600 ppm, synthetic ester also maintains a high breakdown voltage (more than $75 \mathrm{kV}$ ).

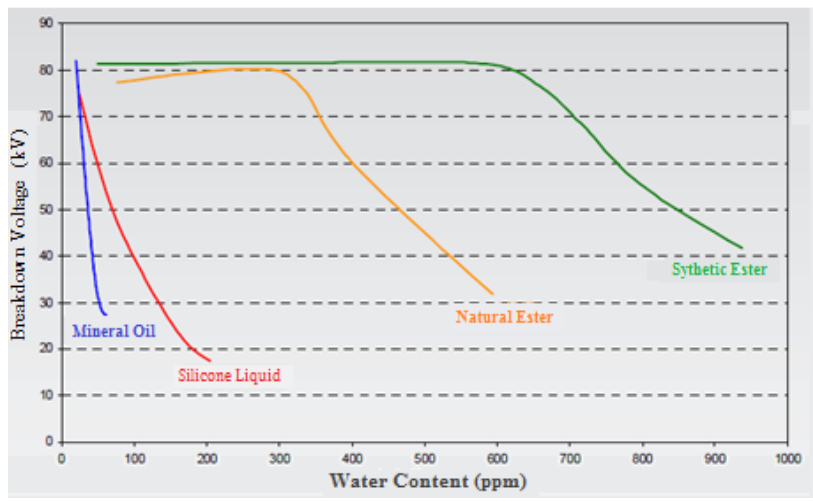

Fig 5 Breakdown Voltage and Water Content at $20{ }^{\circ} \mathrm{C}$

2) Increased water content will cause accelerated aging of the insulation paper.

Various studies show that the insulating paper aging rate has a direct relationship with the water content, the water content of the cellulose every $1 \%$ increase the life of insulating paper will be reduced by $10 \%$. With the aging of cellulose, it will release water, which would aggravate the aging process. The good water absorption capacity of synthetic ester will reduce the water content of insulating paper, so it will slow down the speed of aging. 
3) Increased water content will form bubbles easily.

Bubble will decline the electrical performance of insulating oil. According to IEC 60076-14 standard, the temperature of bubbles production is directly related to the water content of the cellulose. Under overload conditions, the temperature of iron core wrapped by insulation paper will rise. If the water content of insulating paper is $2.6 \%$, the bubble generation temperature is $130{ }^{\circ} \mathrm{C}$. If the water content is $1.1 \%$, the bubble generation temperature is $165^{\circ} \mathrm{C}$. Since the synthetic ester can ensure insulation paper drier, the safety threshold is higher under overload condition.

4) Increased water content will increase the risk of releasing free water from the oil

In the transformer cooling process from working temperature to environment temperature, it is possible to release water from transformer fluid which depends on the water content saturation value. The value of synthetic ester is high, which means it is not easy to achieve its saturation limit and it is difficult to release free water.

\subsection{Lubrication Property Comparison}

Lubrication property is very important to transformer which works continuously and efficiently. If the transformer fluid does not reduce the friction of metal, it will cause the transformer premature failure. For example: transformer fluid pump requires effective lubrication to prevent the occurrence of breakdown. It can be got the lubricating effect of the different transformer fluid by Four Ball Wear experiment. The experiment result is shown in Figure 6, the wear value of synthetic ester is the minimum, which means the lubricating effect of synthetic ester is the best.

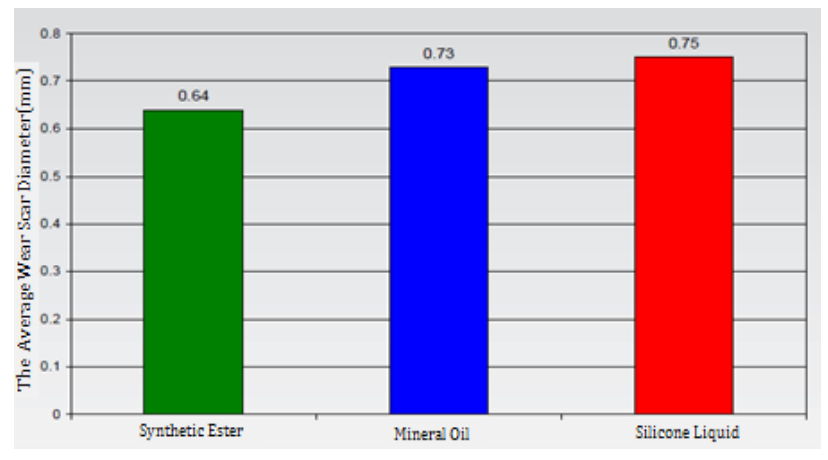

Fig 6 Result of Four Ball Wear Experiment

Synthetic ester shows an excellent performance in terms of moisture resistance and lubrication property. It can absorb more water, and guarantee the reliability of rail transportation equipment effectively.

\section{Low Maintenance}

The average life of the transformer is 30 years, the life and replacement cycle of transformer fluid is influenced by oxidation resistance and aging resistance. If transformer fluid and transformer have the same life, they can be replaced simultaneously.

\subsection{Oxidation stability Comparison}

Oxidation will accelerate the aging speed of transformer fluid, and cause deposition and degeneration of basic performance, high temperature will intensify the oxidation. The method to compare different oxidation stability of transformer fluid is the pressurized vessel oxidation test in ASTMD 2112 standard. The principle is to detect the oxygen consumption time to check the oxidation of oil. When the pressure drop is constant, the longer time-consuming means the stronger oxidation stability of transformer fluid. Three kinds of transformer fluid test result is shown in Figure 7. Oxygen consumption time of synthetic ester is the longest in the test, which means the oxidation stability of synthetic ester is high. However, mineral oil and silicone liquid are relatively poor.

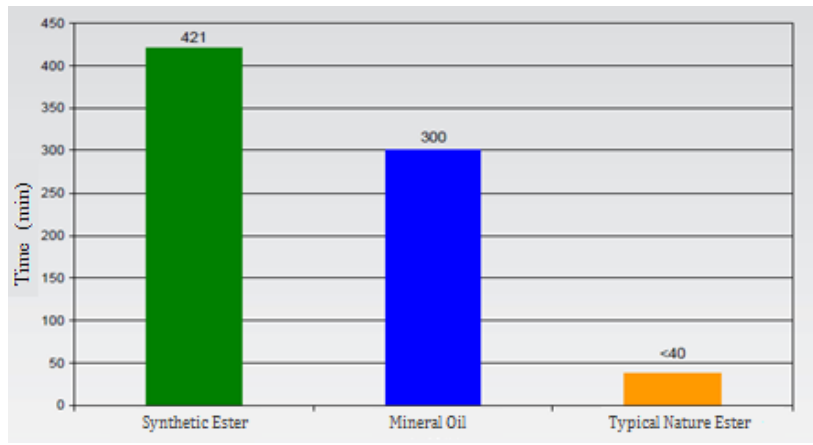

Fig 7 Three Kinds of Transformer Fluid Test Result

\subsection{Aging Resistance Comparison}

The acidity is a key indicator of transformer fluid aging. Transformer is experimented seal aging experiment which is made of common materials (such as copper) and using synthetic ester as transformer fluid. After more than a year of experiment period, the acidity of the synthetic ester is shown in Figure 8. Under the conditions of $125^{\circ} \mathrm{C}$, 
$150{ }^{\circ} \mathrm{C}$ and $160{ }^{\circ} \mathrm{C}$, the acidity of the synthetic ester are kept below prescribed limits in IEC 61203, which means the aging resistance of synthetic ester is very good. Meanwhile, viscosity, density and ignition parameters were monitored during the experiment period, and these parameters are not changed a lot. In addition, synthetic ester did not produce corrosive sulfur and other sediments during the experiment period. All these illustrate that the stability of synthetic ester is good, it may extend the transformer fluid replacement cycle.

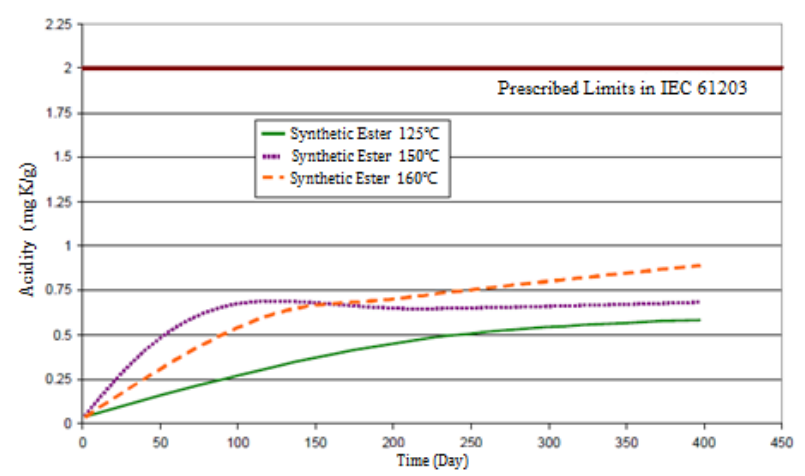

Fig 8 Acidity Change in Seal Aging Experiment

Therefore, oxidation resistance and aging resistance of synthetic esters are excellent, synthetic ester could improve the stability of the transformer, extend the maintenance cycle and reduce maintenance costs.

\section{Conclusions}

Synthetic ester is an excellent performance insulating oil, including fire safety, environmental protection and good moisture resistance. Compared with the other two transformer fluid, synthetic ester has a significant advantage to extend the life of transformer and reduce maintenance costs. Internationally, Siemens, ABB, Bombardier, Alstom and other well-known manufacturer of rail transportation equipment have been widely used synthetic ester, they have rich experience. Speed of 350 $\mathrm{km}$ Chinese standard EMU also chose synthetic ester as transformer fluid. After a comprehensive analysis, synthetic ester is the best choice as transformer fluid, and it could completely replace mineral oil and silicone liquid. As people pay more attention to safety, environmental protection, synthetic ester will be the preferred transformer fluid of electric locomotives, EMUs and suburban rail transport vehicles.

\section{References}

1. ZHANG Shuguang .CRH2 EMU [M] Beijing: China Railway Publishing House, 2008: 167.

2. ZHANG Shuguang .CRH5 EMU [M] Beijing: China Railway Publishing House, 2008: 248.

3. Liu Hong. High Speed Railway and EMU Traction Transformer Oil Analysis[J]. Petroleum Products Application Research, 2010 No.6.

4. ASTM D2112 - 01a (2007) Standard Test Method for Oxidation Stability of Inhibited Mineral Insulating Oil by Pressure Vessel[S].

5. IEC 60076-14. Liquid-immersed power transformers using high-temperature insulation materials $[\mathrm{S}]$.

6. IEC 61039. Classification of insulating liquids[S].

7. IEC 61203. Synthetic organic esters for electrical purposes; guide for maintenance of transformer esters in equipment[S].

8. ISO 2512. Furfural for industrial use; Determination of total carbonyl compounds; Volumetric method[S].

9. ISO 2719. Petroleum products and lubricants; determination of flash point; Pensky-Martens closed cup method[S]. 